\title{
Large nonuniform bending of beams with compressible stored energy functions of polynomial-type
}

\author{
Luca Lanzoni ${ }^{1}$, Angelo Marcello Tarantino ${ }^{2}$
}

January 7,2021

${ }^{1}$ DIEF, Università di Modena e Reggio Emilia, via P. Vivarelli 10, 41125, Modena, Italy, e-mail: luca.lanzoni@unimore.it,

${ }^{2}$ DIEF, Università di Modena e Reggio Emilia, via P. Vivarelli 10, 41125, Modena, Italy, e-mail: angelomarcello.tarantino@unimore.it

\begin{abstract}
The large bending of beams made with complex materials finds application in many emerging fields. To describe the nonlinear behavior of these complex materials such as rubbers, polymers and biological tissues, stored energy functions of polynomial-type are commonly used. Using polyconvex and compressible stored energy functions of polynomial-type, in the present paper the equilibrium problem of slender beams in the fully nonlinear context of finite elasticity is formulated. In the analysis, the bending is considered nonuniform, the complete three-dimensional kinematics of the beam is taken into account and both deformation and displacement fields are deemed large. The governing equations take the form of a coupled system of three equations in integral form, which is solved numerically through an iterative procedure. Explicit formulae for displacements, stretches and stresses in every point of the beam, following both Lagrangian and Eulerian descriptions, are derived. By way of example, a complete analysis has been performed for the Euler beam.
\end{abstract}

Keywords: Finite elasticity; Hyperelasticity; Equilibrium; Beam; Bending moment, Stored energy function.

\section{Introduction}

Today we attend to an extraordinary evolution of technical applications based on the finite bending of beams. For example, electric sensors have recently been applied to human motion monitoring and human-machine interaction [1], 
[2]. These sensors are integrated into flexible beams, offering some advantages such as extreme durability and high reproducibility [3]. Piezoelectric actuators are used for their excellent guiding accuracy during bending of beams. These devices are capable of converting small change in length into a large vertical displacement [4]. Large bending actuators are also made using shape memory alloys (SMA). In this case, a contractile wire, usually in nickel-titanium, is applied on the surface of a flexible beam or strip [5]. Robots based on extremely compliant components constitute an emerging field (so called soft robots) [6]. They are used to produce robotic tentacles [7], to simulate artificial systems [8], self-propulsive robotic fishes [9], human hands [10] and other gripper tools [11]. These high-tech devices can be stimulated to move by changes, for example, in the intensity of the light [12], environmental humidity [13] or electric field [14], etc.

The analysis of bending beams in the context of finite elasticity is considerably complicated by the intrinsic nonlinearities in the mathematical formulation due to the kinematics, the constitutive law and the equilibrium conditions. The main contributions to formulation and resolution of this equilibrium problem are briefly outlined in the following.

The large uniform bending of a plate was first studied by Seth [15]. Based on the semi-inverse approach, he assumed the deformed configuration of the plate like a short circular cylindrical shell, keeping valid the Bernoulli-Navier hypothesis for cross sections. In addition, he assumed a plane strain condition along the axis of the cylinder and a linear constitutive relationship.

The uniform flexion of an elastic block has been investigated by Rivlin [16]. The bending transforms the block into a cylinder with the base in the shape of a circular crown sector. No displacements along the axis of the cylinder have been considered, making the problem as a matter of fact two-dimensional. Rivlin also showed that, in the case of a neo-Hookean material, the surface tractions necessary to induce the hypothesized deformed configuration are statically equivalent to two equal and opposite couples acting at the end faces. Several papers based on Rivlin's solution can be found in Literature (see, e.g., [17], [18], [19] and [20]).

A closed-form solution of a compressible block made of Hencky material has been obtained by Bruhns et al. [21], giving explicit relationships for the bending angle and bending moment as functions of the circumferential stretch. Haughton [22] considered Neo-Hookean and Ogden materials, while Triantafyllidis [23] assumed incrementally linear constitutive relationships to reproduce un elasticplastic behavior. A similar study was carried out by Coman and Destrade [24] and then extended to layered solids by Roccabianca et al. [25].

By passing from a block to a beam a wide amount of studies dealing with large deflections can be found in the Literature for this special structural element under several loading and clamping conditions. A significant part of these studies is based on the solution of the Elastica according to the well-known Euler-Bernoulli law for bending ( $c f$. Love [26]). Shield [27] investigated the problem of a beam under pure bending by assuming large displacements but small strains. In particular, he retrieves the Lamb's solution [28] for the deflec- 
tion of the middle surface of the beam.

After some numerical studies about a cantilever beam subjected at its free edge to a concentrated vertical load, Wang et al. [29] proposed a straightforward numerical method based on the finite differences to solve the equilibrium problem of beams under different load distributions. A comprehensive review on the application of the Elastica can be found in the book by Frisch-Fay [30]. Later, the same subject has been reconsidered by Wang [31], confirming the validity of the Newton-Raphson numerical method in solving the transcendental equations governing the problem, and by Holden [32], who solved the problem through a fourth-order Runge-Kutta procedure. However, in these works, a linear relationship between the curvature and the bending moment has been adopted.

In the framework of Finite Element Methods (FEM) for nonlinear analysis of structures, many works concerning the large displacements and large rotations of beams have been carried out. As an example, Bathe and Bolourchi [33] reported a Lagrangian formulation based on incremental equilibrium equations and a proper decomposition of stresses and strains. Cubic interpolating functions are assumed to describe the displacement field related to bending. A straightforward parametrization of the equation of motion suitable for FEM has been proposed by Simo [34]. In [34], the deformed configuration of a beam is completely described by an orthogonal matrix, from which both the rigid rotations of cross sections and the position of the centroids can be inferred. In addition, in this work, it is shown that the formulation reported by Reissner [35] is exactly retrieved when a plane problem is considered. After the paper by Reissner [36], a lot of studies about the problem of beams under finite displacements have been carried out (e.g., [37], [38], [39], [40], [41] and [42]). However, in these works, neglecting the quadratic part of the Green-de Saint Venant strain tensor, small strains are considered and a linear constitutive relationship is adopted.

In all the above quoted works relating to the bending of blocks and beams under finite displacements, the pure deformation of cross sections has been completely neglected. In this way, the modeling of the problem is simplified substantially, since the displacement field is assumed to be plane, renouncing to describe a phenomenon which in reality is purely three-dimensional. Specifically, the transverse deformation, always coupled with the longitudinal inflexion of a solid, is known as anticlastic effect.

Recently a realistic structural model has been proposed for the large uniform bending of beams by Lanzoni and Tarantino [43]. Following a semi-inverse approach, a three-dimensional kinematic model, where the longitudinal bending is accompanied by the transversal anticlastic deformation of cross sections, has been formulated. The theoretical model proposed in [43] has duly been verified through both numerical analyses and experimental campaigns. The numerical analyses are based on FEM simulations, whereas a test equipment prototype has been designed and manufactured for the large bending of slender beams [44], [45], [46] and [47]. In general, numerical and experimental analyses have provided results that are consistent with those given by the theoretical analysis 
[48].

In view of the many technical applications that can be conducted with this new structural model, the need to generalize the adopted constitutive law has emerged. With this regard, in the present paper the equilibrium problem for beams in the context of finite elasticity has been completely reformulated for a wide class of materials whose stored energy function takes a polynomial expression. For this class of materials, the stored energy function is expressed as a power sum of the principal stretches which satisfies the polyconvexity conditions. In Literature, this density function has been extensively applied to model the nonlinear behavior of complex materials such as rubberlike solids, polymers and biological tissues [49], [50] and [51].

The paper is organized as follows. The kinematic model of inflexed beams with variable curvature is recalled in Section 2. Using a semi-inverse approach, the displacement field derived from the kinematic model contains four unknown functions, which are evaluated in Section 3 by imposing equilibrium conditions. The governing equations constitute a coupled system of nonlinear equations in integral form, which is solved numerically through an iterative procedure. Considering the Euler beam, a numerical application has been performed in Section 4. As the load multiplier grows, the deformed configurations assumed by the beam have been shown and, for the cross section subject to the maximum bending moment, stretches and stresses have been plotted. The Conclusions, delivered in Section 5, close the paper.

\section{Preliminaries, recalls and problem position}

Let us consider a hyperelastic body composed of a homogeneous, isotropic and compressible material ${ }^{1}$ having the shape of a rectangular parallelepiped. Reference is made to a Cartesian coordinate system $\{O, X, Y, Z\}$, with the origin $O$ placed in the centroid of the end cross section, as shown in Fig. 1a. Thus, the body can be identified with the closure of the following regular region:

$$
\mathcal{B}=\left\{(X, Y, Z) \mid-\frac{B}{2}<X<\frac{B}{2},-\frac{H}{2}<Y<\frac{H}{2}, 0<Z<L\right\},
$$

of the three-dimensional Euclidean space $\mathcal{E}$. The symbols $B, H$ and $L$ respectively denote the width, height and length of the body. As is typical in the case of beams, the length $L$ is predominant on the both transverse dimensions $B$ and $H$. As shown in Fig. 1, three-dimensional beams vertically inflexed are considered. Although the formulation will be developed for beams with a rectangular cross section, it can readily extended to beams with a generic cross section provided that the symmetry with respect to $Y$ axis is maintained.

The undeformed configuration $\overline{\mathcal{B}}$ of the beam is assumed as the reference configuration, whereas the deformed configuration is given by the deformation

\footnotetext{
${ }^{1}$ It should be kept in mind that the internal constraint of incompressibility, especially in the case of large deformations, can significantly affect the shape assumed by an inflexed body $[52]$.
} 


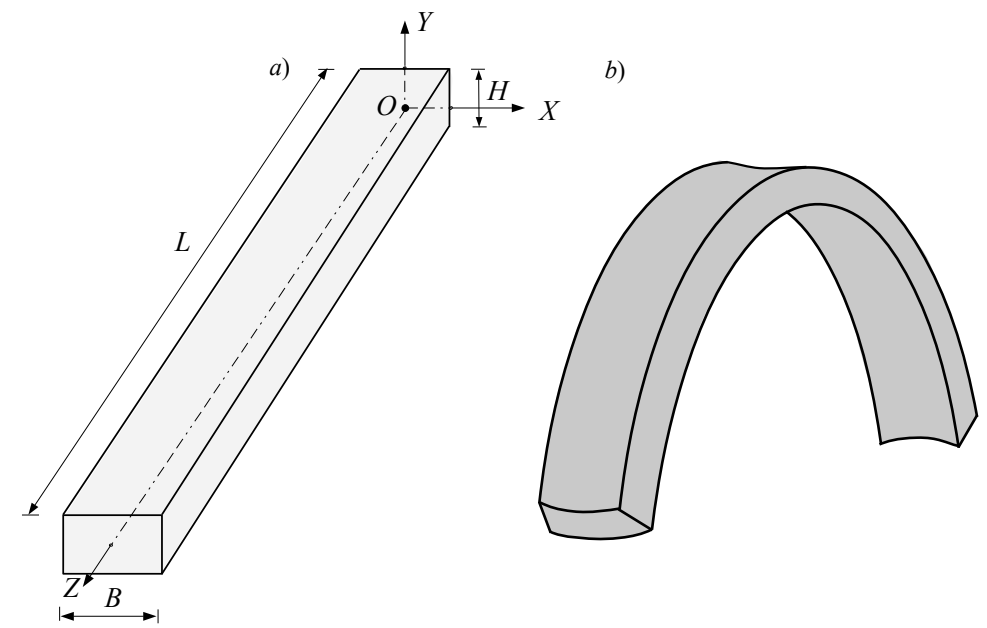

Figure 1: Prismatic slender beam $\overline{\mathcal{B}}$. (a) Undeformed configuration. (b) Deformed configuration.

$\mathbf{f}: \overline{\mathcal{B}} \rightarrow \mathcal{V},{ }^{2}$ that is a smooth enough, injective and orientation-preserving (in the sense that $\operatorname{det} \mathbf{D f}>0$ ) vector field. The deformation of a generic material point $P$ belonging to $\overline{\mathcal{B}}$ can be expressed by the well-known relationship

$$
\mathbf{f}(P)=\mathbf{s}(P)+\mathbf{i d}(P)
$$

where $\mathbf{i d}(P)$ and

$$
\mathbf{s}(P)=u(P) \mathbf{i}+v(P) \mathbf{j}+w(P) \mathbf{k}
$$

are the position and displacement vectors of the point $P$. In the vectorial equation (2), functions $u(P), v(P)$ and $w(P)$ are the scalar components of $\mathbf{s}(P)$, whereas $\mathbf{i}, \mathbf{j}$ and $\mathbf{k}$ are the unit vectors. In the sequel, an Eulerian coordinate system $\{O, x, y, z\}$ is also used (cf. Fig. 2).

To derive the displacement field of an inflexed beam under variable curvature a semi-inverse approach, which involves the definition of a kinematic model, is followed. This model is based on the following three basic hypotheses.

1. In the bending of the beam, cross sections maintain their planarity (BernoulliNavier hypothesis). That is, plane cross sections, orthogonal to $Z$ axis (cf. Fig. 1), remain as such after the beam has been inflexed.

2. Due to the longitudinal inflexion also cross sections are transversely inflexed, but with opposite curvature (anticlastic effect). This transversal inflexion is assumed to occur with constant curvature (cf. Fig. 2c).

3. Slender beams with compact cross sections are considered.

\footnotetext{
${ }^{2} \mathcal{V}$ is the vector space associated with the Euclidean space $\mathcal{E}$.
} 


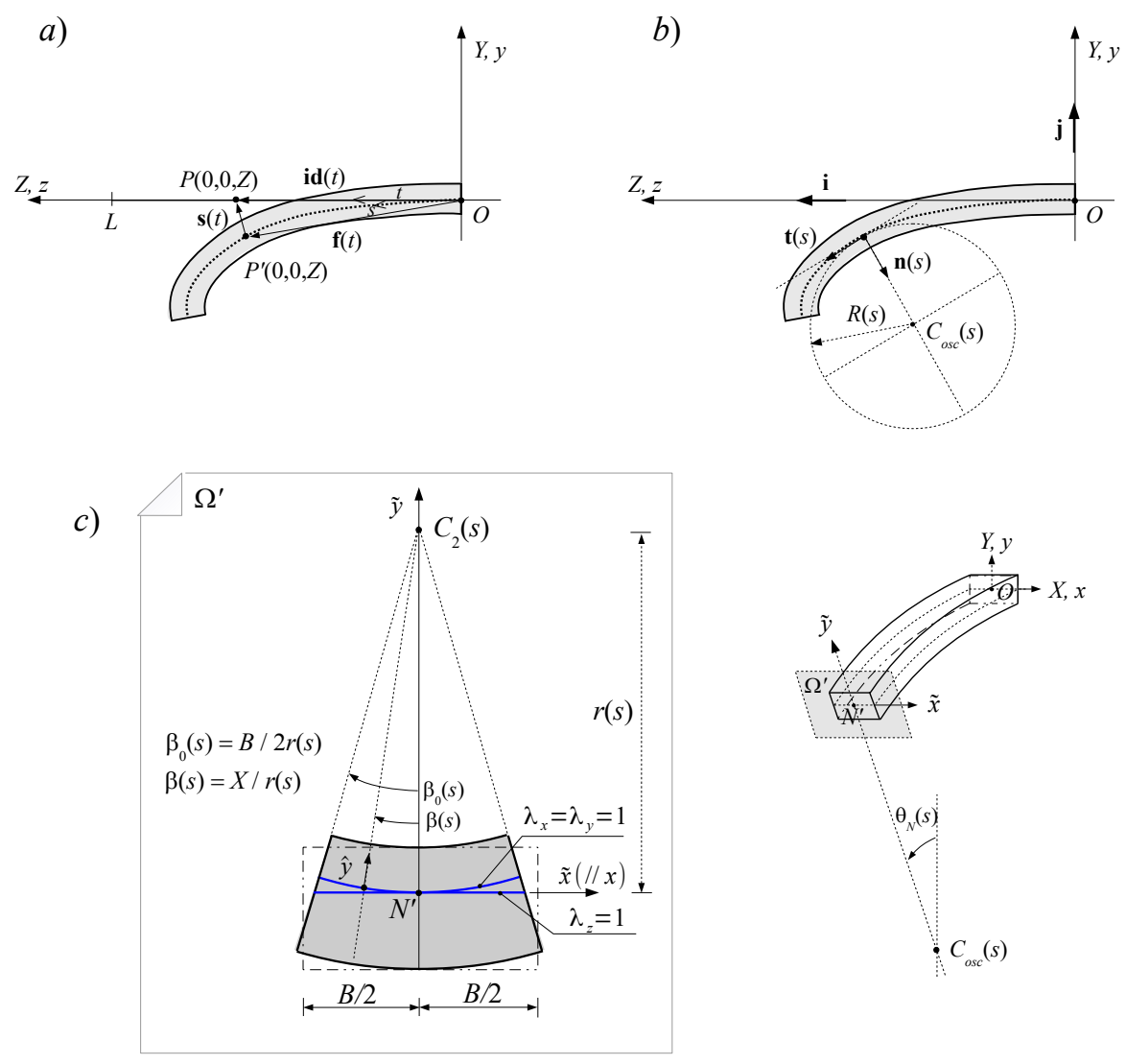

Figure 2: Deformation of a beam. (a) Longitudinal deformation in the vertical plane $X=0$. (b) Longitudinal radius of curvature $R(s)$. (c) Transversal deformation of the generic cross section $\Omega$.

The first assumption, which is very popular in the linear mechanics of beams under pure bending, predicts the conservation of the planarity of cross sections after the deformation. For beams that satisfy the third assumption, the reliability of such hypothesis in nonlinear theory has recently been checked both from the experimental and numerical points of view in [44], [45] and [48]. The second hypothesis also becomes acceptable when beams satisfy the third hypothesis [48].

Based on the three previous hypotheses, the displacement field is derived as the outcome of the coupled effects generated by both longitudinal and transversal curvatures [43]: 


$$
\left\{\begin{array}{l}
u=-X+r(s) e^{-\frac{Y}{r(s)}} \sin \frac{X}{r(s)} \\
v=v_{N}(s)-Y+r(s)\left[1-e^{-\frac{Y}{r(s)}} \cos \frac{X}{r(s)}\right] \cos \theta_{N}(s) \\
w=w_{N}(s)+r(s)\left[1-e^{-\frac{Y}{r(s)}} \cos \frac{X}{r(s)}\right] \sin \theta_{N}(s),
\end{array}\right.
$$

where $v_{N}(s)=v(0,0, Z)$ and $w_{N}(s)=w(0,0, Z)$ are the displacement components of the centroid of the generic cross section, whereas $\theta_{N}(s)=\theta(0,0, Z)$ represents its rotation. The function $r(s)$ denotes the anticlastic radius (cf. Fig. 2c). As shown in [48], in the case of slender beams, the beam axis does not undergo variations in length $\left(\lambda_{Z}=1\right)$. Thus, although the beam is not, its axis can be considered inextensible. Consequently, $Z=s$, where $s$ is the curvilinear abscissa measured in the deformed configuration (cf. Fig. 2a).

Using (1) and (3), the components of the deformation gradient $\mathbf{F}$ can be expressed $\mathrm{as}^{3}$

$$
[\mathbf{F}]=\left[\begin{array}{ccc}
\lambda_{X} \cos \beta(s) & -\lambda_{Y} \sin \beta(s) & 0 \\
\lambda_{X} \sin \beta(s) \cos \theta_{N}(s) & \lambda_{Y} \cos \beta(s) \cos \theta_{N}(s) & -\lambda_{Z} \sin \theta_{N}(s) \\
\lambda_{X} \sin \beta(s) \sin \theta_{N}(s) & \lambda_{Y} \cos \beta(s) \sin \theta_{N}(s) & \lambda_{Z} \cos \theta_{N}(s)
\end{array}\right] .
$$

Given the polar decomposition theorem, $\mathbf{F}=\mathbf{R U}$, it is immediate to write the deformation gradient (4) as product of the rotation tensor $\mathbf{R}$ by the stretch tensor $\mathbf{U}$, where ${ }^{4}$

$$
[\mathbf{R}]=\left[\begin{array}{ccc}
\cos \beta(s) & -\sin \beta(s) & 0 \\
\sin \beta(s) \cos \theta_{N}(s) & \cos \beta(s) \cos \theta_{N}(s) & -\sin \theta_{N}(s) \\
\sin \beta(s) \sin \theta_{N}(s) & \cos \beta(s) \sin \theta_{N}(s) & \cos \theta_{N}(s)
\end{array}\right]
$$

and

$$
[\mathbf{U}]=\left[\begin{array}{ccc}
\lambda_{X} & 0 & 0 \\
0 & \lambda_{Y} & 0 \\
0 & 0 & \lambda_{Z}
\end{array}\right]
$$

On the basis of the hypotheses formulated for the kinematic model, the deformation gradient (4) describes the deformation state of each single point of the beam. The rotation tensor (5) highlights that each point undergoes a longitudinal rotation $\theta_{N}(s)$, which is the same for the entire cross section, and a transversal rotation $\beta(s)=X / r(s)$, within the cross section, that depends on the variable $X$ (cf. Fig. 2c). The stretch tensor (6) characterizes the pure deformations. It is diagonal because the reference system $\{O, X, Y, Z\}$ is principal for the deformation state derived from (3). The principal stretches $\lambda_{X}, \lambda_{Y}$ and $\lambda_{Z}$ in (6) have the following expressions:

\footnotetext{
${ }^{3}$ The expression of $r(s)$ will be evaluated below by a constitutive relationship as a function of the local value of the longitudinal radius $R(s)$, consequently it does not depend on the variations of $R(s)$ along the beam axis. Therefore, according to a classical local constitutive theory, $\frac{d r}{d s}=0$.

${ }^{4} \mathbf{R}$ is a proper orthogonal tensor, $\mathbf{Q}^{\mathrm{T}}=\mathbf{Q}^{-1}$, with $\operatorname{det} \mathbf{Q}=1$. $\mathbf{U}$ is a positive definite and symmetric tensor, $\mathbf{U n} \cdot \mathbf{n}>0$ for $\forall \mathbf{n}-\{\mathbf{o}\}$ and $\mathbf{U}=\mathbf{U}^{\mathbf{T}}$.
} 


$$
\left\{\begin{array}{l}
\lambda_{X}=\lambda_{Y}=e^{-\frac{Y}{r(s)}} \\
\lambda_{Z}=1+\frac{r(s)}{R(s)}\left[1-e^{-\frac{Y}{r(s)}} \cos \frac{X}{r(s)}\right],
\end{array}\right.
$$

being

$$
\frac{d \theta_{N}}{d s}=\frac{1}{R(s)} .
$$

In the previous formula, $R(s)$ denotes the longitudinal radius of curvature of the beam axis (cf. Fig. $2 b$ ).

On the basis of the hypotheses formulated at the beginning of this Section, the nonlinear displacement and deformation fields for beams subject to variable bending moment have been recalled. In these fields there are four functions that have not yet been determined: The three functions related to the deformed axis of the beam, $v_{N}(s), w_{N}(s)$ and $\theta_{N}(s)$, and the anticlastic radius, $r(s)$. These unknown functions will be evaluated in the next Section by adopting a specific constitutive law and subsequently by imposing the equilibrium conditions.

\section{Constitutive relationships and equilibrium}

Constitutive properties of a hyperelastic material are described by the stored energy function $\omega$. If the function $\omega$ is frame-indifferent, homogeneous and isotropic, then it depends only on the principal invariants $I_{i}$, with $i=1,2$ and 3 , of the left Cauchy-Green strain tensor $\mathbf{B}=\mathbf{F F}^{\mathrm{T}}$ (which coincide with those of the right Cauchy-Green strain tensor $\left.\mathbf{C}=\mathbf{F}^{\mathrm{T}} \mathbf{F}\right)^{5}$

$$
\begin{gathered}
I_{1}=\|\mathbf{F}\|^{2}=\lambda_{X}^{2}+\lambda_{Y}^{2}+\lambda_{Z}^{2}, \\
I_{2}=\left\|\mathbf{F}^{\star}\right\|^{2}=\lambda_{X}^{2} \lambda_{Y}^{2}+\lambda_{X}^{2} \lambda_{Z}^{2}+\lambda_{Y}^{2} \lambda_{Z}^{2}, \\
I_{3}=(\operatorname{det} \mathbf{F})^{2}=\lambda_{X}^{2} \lambda_{Y}^{2} \lambda_{Z}^{2} .
\end{gathered}
$$

With these assumptions, the constitutive law $\left(\mathbf{T}_{R}=\partial \omega / \partial \mathbf{F}\right)$ takes the following form:

$$
\mathbf{T}_{R}=2\left(\frac{\partial \omega}{\partial I_{1}}+I_{1} \frac{\partial \omega}{\partial I_{2}}\right) \mathbf{F}-2 \frac{\partial \omega}{\partial I_{2}} \mathbf{B F}+2 I_{3} \frac{\partial \omega}{\partial I_{3}} \mathbf{F}^{-\mathrm{T}},
$$

where the tensor $\mathbf{T}_{R}$ denotes the (first) Piola-Kirchhoff stress tensor.

Being $\mathbf{B F}=\mathbf{R} \mathbf{U}^{3}$ and $\mathbf{F}^{-\mathrm{T}}=\mathbf{R} \mathbf{U}^{-1}$, the tensorial equation (9) can be rewritten as

$$
\mathbf{T}_{R}=\mathbf{R S}
$$

\footnotetext{
${ }^{5}$ The following notations: $\|\mathbf{A}\|=\left(\operatorname{tr} \mathbf{A}^{\mathrm{T}} \mathbf{A}\right)^{1 / 2}$ for the tensor norm in the linear tensor space $\operatorname{Lin}$ and $\mathbf{A}^{\star}=(\operatorname{det} \mathbf{A}) \mathbf{A}^{-\mathrm{T}}$ for the cofactor of the tensor $\mathbf{A}$ (if $\mathbf{A}$ is invertible) are used.
} 
From the kinematic model, the tensors $\mathbf{R}$ and $\mathbf{U}$ are known (see (5) and (6)). Being the tensor $\mathbf{U}$ diagonal also the tensor $\mathbf{S}$ in (10) is such

$$
[\mathbf{S}]=\left[\begin{array}{ccc}
S_{X} & 0 & 0 \\
0 & S_{Y} & 0 \\
0 & 0 & S_{Z}
\end{array}\right],
$$

where

$$
S_{J}=2\left(\frac{\partial \omega}{\partial I_{1}}+I_{1} \frac{\partial \omega}{\partial I_{2}}\right) \lambda_{J}-2 \frac{\partial \omega}{\partial I_{2}} \lambda_{J}^{3}+2 I_{3} \frac{\partial \omega}{\partial I_{3}} \frac{1}{\lambda_{J}}, \quad \text { for } J=X, Y, Z .
$$

Equilibrium requires that the following vectorial equation be satisfied locally:

$$
\operatorname{Div} \mathbf{T}_{R}+\mathbf{b}=\mathbf{o} .
$$

In the absence of body forces $\mathbf{b}$ and computing the scalar components of the material divergence of $\mathbf{T}_{R}$, the vectorial equation (11) provides the following system composed of three partial differential equations:

$$
\left\{\begin{array}{l}
-S_{X} \frac{1}{r(s)} \sin \beta(s)+S_{X, X} \cos \beta(s)-S_{Y, Y} \sin \beta(s)=0 \\
S_{X} \frac{1}{r(s)} \cos \beta(s) \cos \theta_{N}(s)+S_{X, X} \sin \beta(s) \cos \theta_{N}(s)+S_{Y, Y} \cos \beta(s) \cos \theta_{N}(s) \\
-S_{Z} \frac{1}{R(s)} \cos \theta_{N}(s)-S_{Z, Z} \sin \theta_{N}(s)=0 \\
S_{X} \frac{1}{r(s)} \cos \beta(s) \sin \theta_{N}(s)+S_{X, X} \sin \beta(s) \sin \theta_{N}(s)+S_{Y, Y} \cos \beta(s) \sin \theta_{N}(s) \\
-S_{Z} \frac{1}{R(s)} \sin \theta_{N}(s)+S_{Z, Z} \cos \theta_{N}(s)=0 .
\end{array}\right.
$$

The derivatives $S_{J, J}=\frac{\partial S_{J}}{\partial J}$, for $J=X, Y, Z$ (no sum) are reported in the Appendix. System (12), governing equilibrium conditions locally, shows an exceptional complexity and, consequently, a practical impossibility of being solved formally. But above all, it must be taken in mind that, since the shape of the displacement field has been assigned a priori through the hypothesized kinematic model, system (12) cannot provide the exact equilibrium solution for all internal points of the beam.

Nevertheless, some correct information can be obtained by imposing the equilibrium in special points of the beam. In particular, the points of the beam axis show a kinematics completely and properly described by the two radii $R(s)$ and $r(s) .{ }^{6}$ With the aim of rewriting the system (12) for the points of the beam axis it is observed that for them ${ }^{7}$

$$
\lambda_{X}=\lambda_{Y}=\lambda_{Z}=1,
$$

\footnotetext{
${ }^{6}$ The radius $r(s)$ in the kinematical model is assessed at the points belonging to the beam axis and then it has been employed to describe the displacement of all other points of the cross section. Therefore, as one moves away from the beam axis, the value of $r(s)$ can become approximate.

${ }^{7}$ The three $S_{J}$ terms are the same because, at the beam axis, stretches and invariants are the same.

The expressions of $S_{X, X}$ and $S_{Z, Z}$ are similar because, on the basis of the kinematic model, it results: $\lambda_{X, X}=I_{1, X}=I_{3, X}=0$ and $\lambda_{Z, Z}=I_{1, Z}=I_{3, Z}=0$. The expression of $S_{Y, Y}$ is different for the dependence on the variable $Y$ of $\lambda_{Y}, I_{1}$ and $I_{3}$.
} 


$$
\begin{gathered}
\lambda_{Y, Y}=-\frac{1}{r(s)}, \quad \lambda_{Z, Z}=0 \\
I_{1}=3, \quad I_{1, X}=0, \quad I_{1, Y}=-\frac{4}{r(s)}+\frac{2}{R(s)}, \quad I_{1, Z}=0 \\
I_{3}=1, \quad I_{3, X}=0, \quad I_{3, Y}=-\frac{4}{r(s)}+\frac{2}{R(s)}, \quad I_{3, Z}=0 \\
S_{X}=S_{Y}=S_{Z}=2 \omega_{1}+4 \omega_{2}+2 \omega_{3} \\
S_{X, X}=2 \omega_{1, X}+4 \omega_{2, X}+2 \omega_{3, X}, \\
S_{Y, Y}=-\frac{2}{r(s)} \omega_{1}+2 \omega_{1, Y}+\left[\frac{4}{R(s)}-\frac{8}{r(s)}\right] \omega_{2}+4 \omega_{2, Y}+\left[\frac{4}{R(s)}-\frac{6}{r(s)}\right] \omega_{3}+2 \omega_{3, Y} \\
S_{Z, Z}=2 \omega_{1, Z}+4 \omega_{2, Z}+2 \omega_{3, Z}
\end{gathered}
$$

and system (12) specializes into $(\beta(s)=0)$

$$
\left\{\begin{array}{l}
S_{X, X}=0 \\
S_{X} \frac{1}{r(s)} \cos \theta_{N}(s)+S_{Y, Y} \cos \theta_{N}(s)-S_{Z} \frac{1}{R(s)} \cos \theta_{N}(s)-S_{Z, Z} \sin \theta_{N}(s)=0 \\
S_{X} \frac{1}{r(s)} \sin \theta_{N}(s)+S_{Y, Y} \sin \theta_{N}(s)-S_{Z} \frac{1}{R(s)} \sin \theta_{N}(s)+S_{Z, Z} \cos \theta_{N}(s)=0 .
\end{array}\right.
$$

Requesting that the stress be zero in the absence of deformation $\left(\lambda_{X}=\lambda_{Y}=\right.$ $\lambda_{Z}=1$ ), it is obtained: $S_{X}=S_{Y}=S_{Z}=0$, and system (14) reduces to

$$
\left\{\begin{array}{l}
S_{X, X}=0 \\
S_{Y, Y} \cos \theta_{N}(s)-S_{Z, Z} \sin \theta_{N}(s)=0 \\
S_{Y, Y} \sin \theta_{N}(s)+S_{Z, Z} \cos \theta_{N}(s)=0 .
\end{array}\right.
$$

Since this system must be solved for any $\theta_{N}(s)$ values, it can be further reduced

$$
\left\{\begin{array}{l}
S_{X, X}=0 \\
S_{Y, Y}=0 \\
S_{Z, Z}=0
\end{array}\right.
$$

System (15) expresses the equilibrium conditions for the points belonging to the beam axis. In particular, the first equation governs the equilibrium along the $X$ axis. The second and third one, in coupled way, govern the equilibrium along the $Y$ and $Z$ axes.

In order to solve the system (15) some (only sufficient) conditions can be immediately identified to have $S_{X, X}=S_{Z, Z}=0$ for $X=Y=0\left(^{8}\right)$

\footnotetext{
${ }^{8}$ Obviously these derivatives can be different from zero for the other points of the cross section.
} 


$$
\omega_{1, X}=\omega_{2, X}=\omega_{3, X}=0, \quad \omega_{1, Z}=\omega_{2, Z}=\omega_{3, Z}=0 .
$$

The second equation, $S_{Y, Y}=0$, provides then an expression that links the anticlastic radius $r(s)$ to the local value of the longitudinal radius of curvature $R(s)$.

In general, system (15), as set up, is able to provide only information on the radius $r(s)$. By inserting the expression for $r(s)$ in the kinematic model, the radius $R(s)$ remains to be determined. In the following other equilibrium conditions will be exploited to evaluate the others unknown functions.

Lagrangian stresses were expressed by the Piola-Kirchhoff stress tensor $\mathbf{T}_{R}$, while the stress measure coherently employed in the spatial configuration is that of Cauchy. The Cauchy stress tensor $\mathbf{T}$ is obtained from the Piola-Kirchhoff stress tensor $\mathbf{T}_{R}$ through the well-known transformation

$$
\mathbf{T}_{R}=\mathbf{T} \mathbf{F}^{\star},
$$

which given (4), (5), (6) and (10) provides the following components of the Cauchy stress tensor $\left(S=S_{X}=S_{Y}, \lambda=\lambda_{X}=\lambda_{Y}\right)$ :

$$
\begin{gathered}
\left(\lambda^{2} \lambda_{Z}\right)[\mathbf{T}]= \\
{\left[\begin{array}{ccc}
S \lambda & 0 & 0 \\
0 & S \lambda \cos ^{2} \theta_{N}(s)+S_{Z} \lambda_{Z} \sin ^{2} \theta_{N}(s) & \left(S \lambda-S_{Z} \lambda_{Z}\right) \sin \theta_{N}(s) \cos \theta_{N}(s) \\
0 & \left(S \lambda-S_{Z} \lambda_{Z}\right) \sin \theta_{N}(s) \cos \theta_{N}(s) & S \lambda \sin ^{2} \theta_{N}(s)+S_{Z} \lambda_{Z} \cos ^{2} \theta_{N}(s)
\end{array}\right],}
\end{gathered}
$$

being $\mathbf{F}^{\star}=\left(\lambda^{2} \lambda_{Z}\right) \mathbf{R} \mathbf{U}^{-1}$ and $\left(\lambda^{2} \lambda_{Z}\right) \mathbf{T}=\mathbf{R} \mathbf{S} \mathbf{U} \mathbf{R}^{T}$. The tensor $\mathbf{T}$ is symmetric. The matrix (17) can be rewritten in diagonal form by evaluating its eigenvalues. The resolution of the characteristic polynomial allows the determination of the principal Cauchy stresses

$$
[\mathbf{T}]=\left[\begin{array}{ccc}
\frac{S}{\lambda \lambda Z} & 0 & 0 \\
0 & \frac{S}{\lambda \lambda_{Z}} & 0 \\
0 & 0 & \frac{S_{Z}}{\lambda^{2}}
\end{array}\right]
$$

where

$$
\begin{gathered}
\mathrm{T}_{1}=\mathrm{T}_{2}=\frac{S}{\lambda \lambda_{Z}}=\frac{2}{\lambda_{Z}}\left[\omega_{1}+\left(\lambda^{2}+\lambda_{Z}^{2}\right) \omega_{2}+\lambda^{2} \lambda_{Z}^{2} \omega_{3}\right], \\
\mathrm{T}_{3}=\frac{S_{Z}}{\lambda^{2}}=\frac{2}{\lambda^{2}}\left[\lambda_{Z} \omega_{1}+2 \lambda^{2} \lambda_{Z} \omega_{2}+\lambda^{4} \lambda_{Z} \omega_{3}\right] .
\end{gathered}
$$

Since Cauchy stresses are Eulerian functions, in the above formulae it is more appropriate to use the Eulerian stretches derived from (7) [43], [48]

$$
\left\{\begin{array}{l}
\lambda_{x}=\lambda_{y}=\frac{1}{\cos \beta(s)}\left[1-\frac{y-v_{N}(s)}{r(s) \cos \theta_{N}(s)}\right] \\
\lambda_{z}=1+\frac{1}{R(s)} \frac{y-v_{N}(s)}{\cos \theta_{N}(s)} .
\end{array}\right.
$$

The principal directions of stress are the eigenvectors associated with these eigenvalues. The principal direction corresponding to the eigenvalue $T_{3}$ is the 
unit vector orthogonal to the plane $\Omega^{\prime}$ (cf. Fig. 2c) with components $(0$, $\left.-\sin \theta_{N}(s), \cos \theta_{N}(s)\right)$. The others two eigenvectors are any two unit vectors orthogonal to each other and belonging to the plane $\Omega^{\prime}$.

To move forward in the problem formulation it is now necessary to define the constitutive relationship. The use of stored energy function of polynomial-type is probably the most general and popular approach to describe the nonlinear constitutive behavior of complex materials such as rubbers, polymers and biological tissues. In this case, as suggested by Ogden, the first two terms of the stored energy function can be expressed as sum of powers of principal stretches [49], [50], [51] ${ }^{9}$

$$
\begin{gathered}
\tilde{\omega}\left(\lambda_{1}, \lambda_{2}, \lambda_{3}\right)=\sum_{i=1}^{L} A_{i}\left(\lambda_{1}^{\alpha_{i}}+\lambda_{2}^{\alpha_{i}}+\lambda_{3}^{\alpha_{i}}\right)+\sum_{j=1}^{M} B_{j}\left[\left(\lambda_{1} \lambda_{2}\right)^{\beta_{j}}+\left(\lambda_{1} \lambda_{3}\right)^{\beta_{j}}+\left(\lambda_{2} \lambda_{3}\right)^{\beta_{j}}\right] \\
+\Gamma\left(\lambda_{1} \lambda_{2} \lambda_{3}\right)
\end{gathered}
$$

where $\alpha_{1}>\alpha_{2}>\ldots>\alpha_{L} \geq 1, \beta_{1}>\beta_{2}>\ldots>\beta_{M} \geq 1$, and $A_{i}, B_{j}$ are positive constants. $\Gamma\left(\lambda_{1} \lambda_{2} \lambda_{3}\right)$, with $\lambda_{1} \lambda_{2} \lambda_{3}=\operatorname{det} \mathbf{F}=I_{3}^{1 / 2}=\delta$, is a convex function that satisfies the growth conditions both as $\delta \rightarrow 0^{+}$and $\delta \rightarrow \infty$. If the following inequalities are fulfilled: $\alpha_{1} \geq 2, \frac{1}{\alpha_{1}}+\frac{1}{\beta_{1}} \leq 1$, then, no loss of ellipticity occurs in the equilibrium equations and the existence of solutions of the boundary-value problem is assured in a properly chosen function space [51]. Hereinafter the expression proposed by Ciarlet and Geymonat [53] is chosen for the function $\Gamma(\delta)$

$$
\Gamma(\delta)=c \delta^{2}-d \ln \delta,
$$

where $c$ and $d$ are positive constants.

Note that from (21) the compressible Mooney-Rivlin stored energy function can be obtained as special case $\left(L=M=1, A_{1}=a, B_{1}=b, \alpha_{1}=\beta_{1}=2\right)^{10}$

$$
\omega\left(I_{1}, I_{2}, I_{3}\right)=a I_{1}+b I_{2}+\Gamma(\delta),
$$

and similarly the stored energy function for compressible neo-Hookean materials $\left(L=1, A_{1}=a, B_{j}=0, \alpha_{1}=2\right)$

$$
\omega\left(I_{1}, I_{2}, I_{3}\right)=a I_{1}+\Gamma(\delta) .
$$

A classic constitutive law for hyperelastic materials is usually expressed in terms of derivatives of the stored energy function with respect to the deformation invariants, $\omega_{i}=\frac{\partial \omega}{\partial I_{i}}$, for $i=1,2$ and 3 (cf. (9)), while the variables used in the stored energy function (21) are the three principal stretches $\lambda_{i}$, for $i=1,2$ and 3. To use (21) it is therefore necessary to establish correlation formulae between the derivatives of $\omega$ with respect to the invariants $I_{i}, \omega_{i}=\frac{\partial \omega}{\partial I_{i}}$, and

\footnotetext{
${ }^{9}$ The principal stretches $\lambda_{X}, \lambda_{Y}$ and $\lambda_{Z}$ are renamed respectively with the symbols $\lambda_{1}, \lambda_{2}$ and $\lambda_{3}$.

${ }^{10}$ The compressible Mooney-Rivlin stored energy function was used, for example, in [54], [55], [56] and [57].
} 
those with respect to the stretches $\lambda_{i}, \frac{\partial \tilde{\omega}}{\partial \lambda_{i}}$. Using the chain rule, $\frac{\partial \tilde{\omega}}{\partial \lambda_{i}}=\frac{\partial \omega}{\partial I_{j}} \frac{\partial I_{j}}{\partial \lambda_{i}}$, the following expressions can be written between the two groups of derivatives:

$$
\left\{\begin{array}{l}
\frac{1}{2 \lambda_{1}} \frac{\partial \tilde{\omega}}{\partial \lambda_{1}}=\omega_{1}+\left(\lambda_{2}^{2}+\lambda_{3}^{2}\right) \omega_{2}+\lambda_{2}^{2} \lambda_{3}^{2} \omega_{3} \\
\frac{1}{2 \lambda_{2}} \frac{\partial \tilde{\omega}}{\partial \lambda_{2}}=\omega_{1}+\left(\lambda_{1}^{2}+\lambda_{3}^{2}\right) \omega_{2}+\lambda_{1}^{2} \lambda_{3}^{2} \omega_{3} \\
\frac{1}{2 \lambda_{3}} \frac{\partial \tilde{\omega}}{\partial \lambda_{3}}=\omega_{1}+\left(\lambda_{1}^{2}+\lambda_{2}^{2}\right) \omega_{2}+\lambda_{1}^{2} \lambda_{2}^{2} \omega_{3}
\end{array}\right.
$$

By inverting this system the correlation formulae are obtained

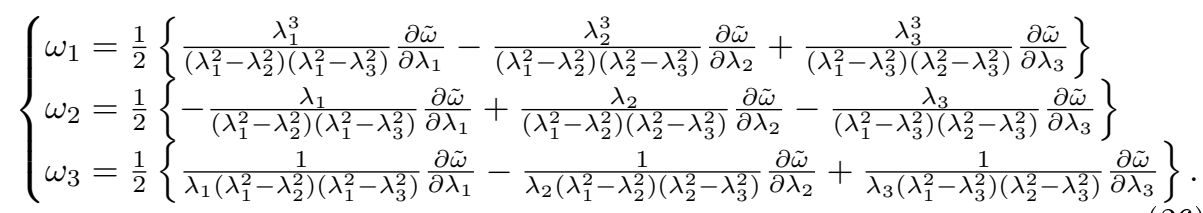

From (21) the derivatives to be introduced into system (26) are

$$
\left\{\begin{array}{l}
\frac{\partial \tilde{\omega}}{\partial \lambda_{1}}=\sum_{i=1}^{L} A_{i} \alpha_{i} \lambda_{1}^{\alpha_{i}-1}+\sum_{j=1}^{M} B_{j} \beta_{j}\left[\lambda_{2}\left(\lambda_{1} \lambda_{2}\right)^{\beta_{j}-1}+\lambda_{3}\left(\lambda_{1} \lambda_{3}\right)^{\beta_{j}-1}\right]+2 c \lambda_{1} \lambda_{2}^{2} \lambda_{3}^{2}-\frac{d}{\lambda_{1}} \\
\frac{\partial \tilde{\omega}}{\partial \lambda_{2}}=\sum_{i=1}^{L} A_{i} \alpha_{i} \lambda_{2}^{\alpha_{i}-1}+\sum_{j=1}^{M} B_{j} \beta_{j}\left[\lambda_{1}\left(\lambda_{1} \lambda_{2}\right)^{\beta_{j}-1}+\lambda_{3}\left(\lambda_{2} \lambda_{3}\right)^{\beta_{j}-1}\right]+2 c \lambda_{1}^{2} \lambda_{2} \lambda_{3}^{2}-\frac{d}{\lambda_{2}} \\
\frac{\partial \tilde{\omega}}{\partial \lambda_{3}}=\sum_{i=1}^{L} A_{i} \alpha_{i} \lambda_{3}^{\alpha_{i}-1}+\sum_{j=1}^{M} B_{j} \beta_{j}\left[\lambda_{1}\left(\lambda_{1} \lambda_{3}\right)^{\beta_{j}-1}+\lambda_{2}\left(\lambda_{2} \lambda_{3}\right)^{\beta_{j}-1}\right]+2 c \lambda_{1}^{2} \lambda_{2}^{2} \lambda_{3}-\frac{d}{\lambda_{3}} .
\end{array}\right.
$$

In the kinematics of the beam illustrated in Section 2, the transversal stretches $\lambda_{1}$ and $\lambda_{2}$ are equal. Then, performing the passage to limit $\lambda_{1} \rightarrow \lambda_{2}$, system (26), with (27), gives $\left(\lambda_{1}=\lambda_{2}=\lambda\right)$

$$
\left\{\begin{array}{c}
\omega_{1}=\frac{1}{4\left(\lambda^{2}-\lambda_{3}^{2}\right)^{2}}\left\{\sum_{i=1}^{N} A_{i} \alpha_{i}\left[\lambda^{\alpha_{i}}\left(\alpha_{i} \lambda^{2}-\left(2+\alpha_{i}\right) \lambda_{3}^{2}\right)+2 \lambda_{3}^{2+\alpha_{i}}\right]+\right. \\
\left.\sum_{j=1}^{M} B_{j} \beta_{j}\left[\left(\lambda \lambda_{3}\right)^{\beta_{j}}\left(\beta_{j} \lambda^{2}-\left(\beta_{j}-2\right) \lambda_{3}^{2}\right)-2 \lambda^{2 \beta_{j}} \lambda_{3}^{2}\right]\right\} \\
\omega_{2}=\frac{1}{4\left(\lambda^{3}-\lambda \lambda_{3}^{2}\right)^{2}}\left\{\sum_{i=1}^{L} A_{i} \alpha_{i}\left[\left(\alpha_{i}-2\right) \lambda^{2+\alpha_{i}}+\alpha_{i} \lambda^{\alpha_{i}} \lambda_{3}^{2}-2 \lambda^{2} \lambda_{3}^{\alpha_{i}}\right]+\right. \\
\left.\sum_{j=1}^{M} B_{j} \beta_{j}\left[2 \lambda^{2+2 \beta_{j}}+\left(\lambda \lambda_{3}\right)^{\beta_{j}}\left(\beta_{j} \lambda_{3}^{2}-\left(\beta_{j}+2\right) \lambda^{2}\right)\right]\right\} \\
\omega_{3}=\frac{1}{4 \lambda^{4} \lambda_{3}^{2}\left(\lambda^{2}-\lambda_{3}^{2}\right)^{2}}\left\{\sum _ { i = 1 } ^ { L } A _ { i } \alpha _ { i } \left[\left(\alpha_{i}-4\right) \lambda^{2+\alpha_{i}} \lambda_{3}^{2}+\left(2-\alpha_{i}\right) \lambda^{\alpha_{i}} \lambda_{3}^{4}+\right.\right. \\
\left.2 \lambda^{4} \lambda_{3}^{\alpha_{i}}\right]+\sum_{j=1}^{M} B_{j} \beta_{j}\left[2 \lambda^{2 \beta_{j}} \lambda_{3}^{4}-4 \lambda^{2+2 \beta_{j}} \lambda_{3}^{2}+\right. \\
\left.\left.\left(\lambda \lambda_{3}\right)^{\beta_{j}}\left(4 \lambda^{4}+\left(\beta_{j}-4\right) \lambda^{2} \lambda_{3}^{2}-\left(\beta_{j}-2\right) \lambda_{3}^{4}\right)\right]\right\}+ \\
c-\frac{d}{2 \lambda^{4} \lambda_{3}^{2}} .
\end{array}\right.
$$

In the even more particular case in which all three stretches are equal, with a further passage to limit, formulae (28) are transformed into $\left(\lambda_{1}=\lambda_{2}=\lambda_{3}=\lambda\right)$

$$
\left\{\begin{array}{c}
\omega_{1}=\frac{1}{16 \lambda^{2}}\left\{\sum_{i=1}^{L} A_{i} \alpha_{i}^{2}\left(2+\alpha_{i}\right) \lambda^{\alpha_{i}}-\sum_{j=1}^{M} B_{j} \beta_{j}^{2}\left(\beta_{j}-2\right) \lambda^{2 \beta_{i}}\right\} \\
\omega_{2}=\frac{1}{16 \lambda^{4}}\left\{\sum_{i=1}^{L} A_{i} \alpha_{i}^{2}\left(2-\alpha_{i}\right) \lambda^{\alpha_{i}}+\sum_{j=1}^{M} B_{j} \beta_{j}^{2}\left(\beta_{j}+2\right) \lambda^{2 \beta_{i}}\right\} \\
\omega_{3}=\frac{1}{16 \lambda^{6}}\left\{\sum_{i=1}^{L} A_{i} \alpha_{i}\left(\alpha_{i}-4\right)\left(\alpha_{i}-2\right) \lambda^{\alpha_{i}}-\right. \\
\left.\sum_{j=1}^{M} B_{j} \beta_{j}\left(\beta_{j}-2\right)\left(\beta_{j}+8\right) \lambda^{2 \beta_{i}}\right\}+c-\frac{d}{2 \lambda^{6}} .
\end{array}\right.
$$


By introducing (28) in (10), Piola-Kirchhoff stresses can be computed for each point of the beam using the stored energy function (21).

Among the constitutive constants involved in (21) a relationship can be established by imposing that, in the absence of deformation, the stress vanishes. By setting $\beta(s)=\theta_{N}(s)=0$ into (5), the stresses $\mathrm{T}_{R, i j}$, with $i \neq j$, for $i, j=$ $1,2,3$, are zero, whereas the diagonal component for $\lambda_{i}=1$ are $^{11}$

$$
\mathrm{T}_{R, 11}=\mathrm{T}_{R, 22}=\mathrm{T}_{R, 33}=\left.2\left(\omega_{1}+2 \omega_{2}+\omega_{3}\right)\right|_{\lambda_{i}=1}=0 .
$$

By inserting in this condition the derivatives (29), written for $\lambda_{i}=1$, the following relationship for the evaluation of the constant $d$ is obtained:

$$
d=\sum_{i=1}^{L} A_{i} \alpha_{i}+2 \sum_{j=1}^{M} B_{j} \beta_{j}+2 c .
$$

Using this expression, at the beam axis, it occurs that $S_{X}=S_{Y}=S_{Z}=0$.

The equilibrium of the points belonging to the beam axis is governed by system (15) and the derivatives $S_{J, J}$, with $J=X, Y$ and $Z$, are specified in the Appendix. In the expressions $S_{J, J}$ there are the partial derivatives of $\omega_{i}$ with respect to the variables $X, Y$ and $Z$, which are evaluated at the points of the beam axis: $X=Y=0$ and $Z=Z$. Therefore, using (7) and (28) and computing partial derivatives, the following nine expressions are obtained:

$$
\begin{gathered}
\omega_{1, X}=\omega_{2, X}=\omega_{3, X}=0 \\
\omega_{1, Y}=\frac{r(s)-2 R(s)}{48 r(s) R(s)}\left\{\sum_{i=1}^{L} A_{i} \alpha_{i}^{2}\left(\alpha_{i}^{2}-4\right)-2 \sum_{j=1}^{M} B_{j} \beta_{j}^{2}\left(\beta_{j}^{2}-3 \beta_{j}+2\right)\right\}, \\
\omega_{2, Y}=-\frac{r(s)-2 R(s)}{48 r(s) R(s)}\left\{\sum_{i=1}^{L} A_{i} \alpha_{i}^{2}\left(\alpha_{i}^{2}-6 \alpha_{i}+8\right)-2 \sum_{j=1}^{M} B_{j} \beta_{j}^{2}\left(\beta_{j}^{2}-4\right)\right\}, \\
\omega_{3, Y}=\frac{r(s)-2 R(s)}{48 r(s) R(s)}\left\{\sum_{i=1}^{L} A_{i} \alpha_{i}\left(\alpha_{i}^{3}-12 \alpha_{i}^{2}+44 \alpha_{i}-48\right)-\right. \\
\left.2 \sum_{j=1}^{M} B_{j} \beta_{j}\left(\beta_{j}^{3}+3 \beta_{j}^{2}-34 \beta_{j}+48\right)+48 d\right\} \\
\omega_{1, Z}=\omega_{2, Z}=\omega_{3, Z}=0 .
\end{gathered}
$$

Since only the derivatives with respect to variable $Y$ are different from zero, the first and third equations of system (15) are identically satisfied

$$
\begin{gathered}
S_{X, X}=2 \omega_{1, X}+4 \omega_{2, X}+2 \omega_{3, X}=0, \\
S_{Z, Z}=2 \omega_{1, Z}+4 \omega_{2, Z}+2 \omega_{3, Z}=0 .
\end{gathered}
$$

\footnotetext{
${ }^{11}$ A similar position has been used, for example, in and [58], [59] and [60].
} 
As already mentioned, the second equation of system (15) can be used to derive the relationship between the two radii $r(s)$ and $R(s)$. Thus, using (13), (32) and (29) evaluated for $\lambda=1$, equation $(15)_{2}$ gives

$$
r(s)=\frac{6 c+d+\sum_{i=1}^{L} A_{i} \alpha_{i}\left(\alpha_{i}-1\right)+\sum_{j=1}^{M} B_{j} \beta_{j}\left(3 \beta_{j}-2\right)}{4 c+\sum_{j=1}^{M} B_{j} \beta_{j}^{2}} R(s),
$$

where the constant $d$ can be calculated with (31). If the radius $r(s)$ takes this expression, the system (15) is fulfilled, although the radius $R(s)$ is still to be determined.

In the special case of a Mooney-Rivlin material, (28), (31) and (33) are reduced respectively to

$$
\begin{gathered}
\omega_{1}=a, \quad \omega_{2}=b, \quad \omega_{3}=c-\frac{d}{2 \lambda^{4} \lambda_{3}^{2}}, \\
d=2(a+2 b+c), \\
r(s)=\frac{a+3 b+2 c}{b+c} R(s) .
\end{gathered}
$$

These expressions coincide with those obtained in [43] and [48].

Once the Piola-Kirchhoff stresses have been evaluated using (21), the corresponding Cauchy stresses can be calculated with the transformation (16) and in particular the principal component $\mathrm{T}_{3}$ can be determined using the $(19)_{2}$. With the component $\mathrm{T}_{3}$, the internal bending moment $m_{x}^{i n t}(s)$ of a generic cross section of the beam in the deformed configuration can be evaluated by integrating the elementary moments generated by the principal Cauchy stress $\mathrm{T}_{3}$ orthogonal to $\Omega^{\prime}$. Thus, using the polar coordinates $(\rho, \beta)$ with pole at the point $C_{2}(s)$ of Fig. $2 \mathrm{c}$, the moment $m_{x}^{i n t}(s)$ can be computed by the following integral:

$$
m_{x}^{i n t}(s)=\int_{-\beta_{0}(s)}^{\beta_{0}(s)} \int_{\rho_{\min }(s)}^{\rho_{\max }(s)} \rho \mathrm{T}_{3}(\rho, \beta) \tilde{y}(\mathrm{~s}) \mathrm{d} \rho \mathrm{d} \beta
$$

where $\tilde{x}(s)=\rho \sin \beta, \tilde{y}(s)=r(s)-\rho \cos \beta$, and

$$
\begin{gathered}
\rho_{\text {min }}(s)=r(s)-\int_{0}^{\frac{H}{2}} \lambda_{Y}(\hat{Y}) d \hat{Y}=r(s) e^{-\frac{H}{2 r(s)}}, \\
\rho_{\max }(s)=r(s)+\int_{\frac{H}{2}}^{0} \lambda_{Y}(\hat{Y}) d \hat{Y}=r(s) e^{\frac{H}{2 r(s)}} .
\end{gathered}
$$

For equilibrium, the internal bending moment (35) must equal the external bending moment $m_{x}^{e x t}(s)$ produced by external loads in correspondence of the same cross section

$$
m_{x}^{i n t}(s)=m_{x}^{e x t}(s)
$$


This relationship depends on the unknown radius $R(s)$ and for this reason we will refer to it as the moment-curvature relationship in the general form. In fact it, derived in the fully nonlinear context of finite elasticity, relates the external bending moment $m_{x}^{\text {ext }}(s)$ with the longitudinal curvature $R(s)^{-1}$, both evaluated along the deformed beam axis. The moment-curvature relationship (36) will be used below to determine the displacement field of the beam axis.

The two displacement components $v_{N}(s)$ and $w_{N}(s)$ allow evaluating the position of the deformed axis of beam, and they can be written in terms of rotation $\theta_{N}(s)$

$$
\begin{gathered}
v_{N}(s)=v_{N}(0)-\int_{0^{+}}^{s} \sin \theta_{N}(\varsigma) d \varsigma, \\
w_{N}(s)=w_{N}(0)-\int_{0^{+}}^{s}\left(1-\cos \theta_{N}(\varsigma)\right) d \varsigma .
\end{gathered}
$$

These formulae are obtained by integrating the following infinitesimal displacements: $d v_{N}=-\sin \theta_{N} d s, d w_{N}=-\left(1-\cos \theta_{N}\right) d s$, generated by the rotation of an infinitesimal and inextensible horizontal element.

At this point, the solving procedure for the nonlinear equilibrium of inflexed beams can be organized into three successive steps.

The first step of procedure concerns the resolution of system composed of equations (36) and (37), whose unknowns are the three kinematic functions $v_{N}(s), w_{N}(s)$ and $\theta_{N}(s)$, that is the displacements and rotations of the beam axis. This system is coupled and nonlinear. It can be solved through the iterative numerical procedure illustrated in Appendix A of [43]. In each single interaction the longitudinal curvature $R(s)^{-1}$ is updated using (8) and consequently also the transversal radius $r(s)$ is modified according to (33). Once the iterative procedure has reached convergence, in addition to the three kinematic functions, the bending moment $m_{x}^{e x t}(s)$ and the two radii $R(s)$ and $r(s)$ are known for the specific case treated.

The second step deals with the determination of the three-dimensional shape assumed by the beam in the deformed configuration. Since functions $v_{N}(s)$, $w_{N}(s), \theta_{N}(s)$ and $r(s)$ are available, the displacements of each point of the beam can be evaluated with (3).

The third step is dedicated to the analysis of stretches and stresses at the points of each cross section of the beam. For this purpose, the Lagrangian expressions (7) and (10) or the Eulerian expressions (20) and (17) can be used.

Following this procedure, the complete solution is obtained. However, it is necessary to keep in mind that the above solution was not obtained through a classic boundary-value problem. But a semi-inverse method has been applied, which hypothesizes the form of the solution by assigning the displacement field unless four unknown functions. Operating in this way inevitably some approximations will be present with respect to the exact solution, the determination of which, however, remains excessively complex. 
To estimate the accuracy of the solution proposed, the vectorial equilibrium equation (11) can be used, checking if it is satisfied locally, that is for each single point of the beam. For this purpose, the three scalar equations derived from (11) are rewritten in dimensionless form, so that their comparison with the scalar zero takes full meaning. Therefore, by evaluating how much these three equations deviate from zero, it is possible to measure the accuracy of the solution obtained. In [52], it has been shown that, for the points belonging to the beam axis, the equilibrium equations are exactly satisfied (see also [46] and [48]). In fact, for these points, the displacement field is correct being it kinematically compatible and equilibrated. When leaving this basic line, equilibrium equations are no longer so well satisfied and approximations grow as the edges of the cross sections are approached. This is because for these points the hypotheses underlying the kinematic model are less accurate. However, as a result of the continuity of the displacement field, it is reasonable to expect the existence of a central core for each cross section where the solution is still acceptable since the equilibrium equations are practically zero. Obviously, a quantitative criterion cannot be defined to estimate the extension of this central core, since the corresponding confidence limits must be necessarily contextualized in the specific problem treated. However, it is important to note that the gap with zero for the points outside the central core decreases considerably as the beam becomes increasingly slender [43].

\section{Numerical application}

As an example of application, the Euler beam is considered. This classic bifurcation problem will be reformulated, and analyzed in all its aspects, on the basis of the beam model developed in this paper. A beam with the following geometrical dimensionless parameters: $H=1, B=2, L=15$ is taken into account. The constitutive behavior of this beam is described by (21), where the series are truncated at the third term $(L=2, M=1)$ and the following exponents: $\alpha_{1}=4, \alpha_{2}=2$ and $\beta_{1}=2$ and dimensionless constitutive constants: $A_{1}=0.1, A_{2}=1, B_{1}=0.1$ and $c=1$ have been chosen [61]. Adopting these parameters, (31) gives $d=4.8$.

As it is well known, immediately after the bifurcation, which occurs when the axial compressive force reaches the Euler load $P_{C R}=\pi^{2} \frac{E J_{X}}{L^{2}}$, the beam assumes one of the two adjacent stable equilibrium configurations. ${ }^{12}$ In these symmetrical configurations, the shape of the beam axis is described by the following expression: $v(Z)=v_{0} \sin \frac{\pi Z}{L}$, where $v_{0}$ is the vertical displacement at $Z=L / 2$. In the classical analysis of the Euler beam, the parameter $v_{0}$ remains undetermined. Otherwise, in the present case study, the three-dimensional shape taken by the beam after bifurcation is completely determined. With reference to the upper deflection curves, Fig. 3a shows the equilibrium post-buckling paths of the Euler beam as the multiplier $\mu$ of $P_{C R}$ increases. In this figure, the beam

\footnotetext{
${ }^{12}$ The symbol $E$ denotes the Young's modulus and $J_{X}$ the moment of inertia of the undeformed cross section with respect to the $X$ axis.
} 


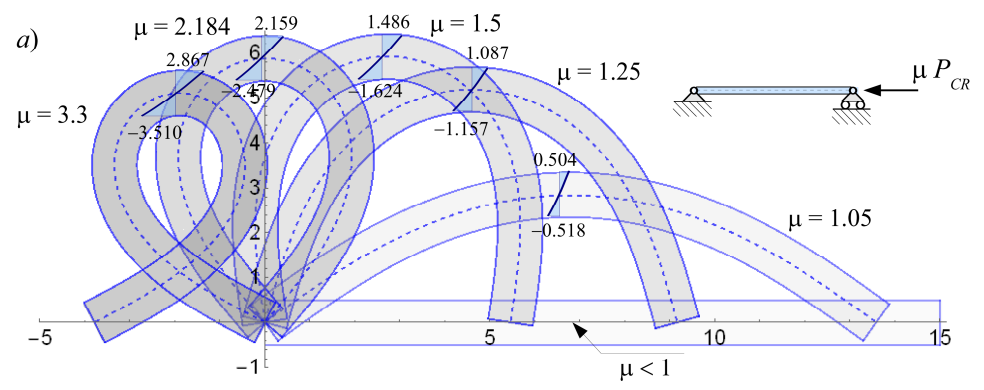

b)
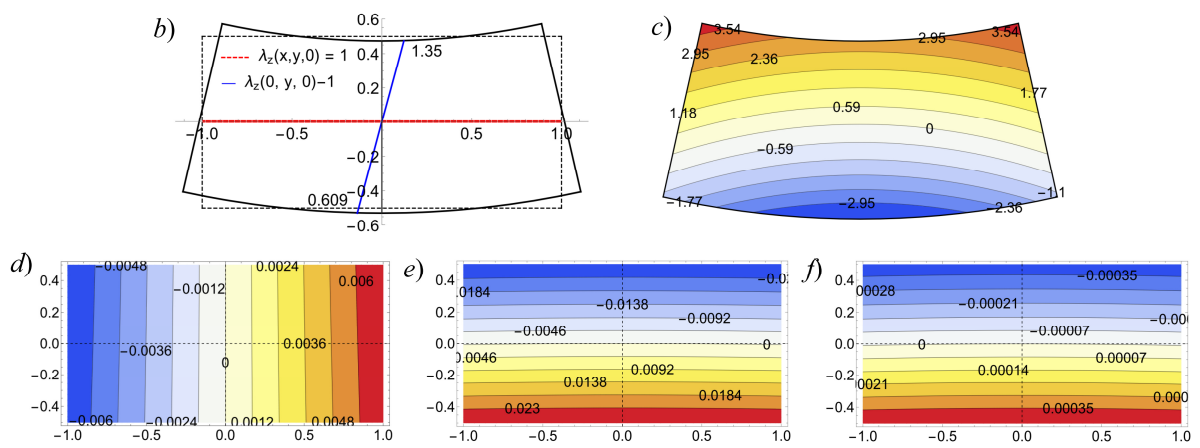

Figure 3: Euler beam inflexed by $\mu P_{C R}$. (a) Deformed beams as the load multiplier $\mu$ varies. Case with $\mu=3.3$. Cross section with maximum bending moment $(s=L / 2)$, for which $m_{x}=949.787, \theta=0, R=13.5298, R^{(1)}=0.0146706$ and $r=45.5093$. (b) Eulerian stretches. Vertical diagram of $\left(\lambda_{z}(0, y, L / 2)-1\right)$ and neutral axis for the deformation $\left(\lambda_{z}(x, y, L / 2)=1\right)$. (c) Cauchy stress component $\mathrm{T}_{3}$. Plot of the equilibrium equations (11). (d), (e) and (f) Equilibrium along the $X, Y$ and $Z$ axis, respectively. 
in the different deformed configurations, as well as the thicknesses, are plotted exactly in scale. As $\mu$ grows, the right sliding support moves towards the left hinge. For $\mu=2.184$, the support has traveled the full length of the beam, reaching the hinge. In this particular situation, the beam axis takes the shape of a drop. After this value of $\mu$, the support exceeds the hinge, and the beam forms a loop.

To apply the numerical procedure exposed at the end of Section 3, the Euler beam has been discretized into 100 sampling nodes. The first-step trial solution has been taken proportional to the initial sinusoidal deflection curve. Up to $\mu=1.5$, the convergence towards the equilibrium solution requires less than 15 iterations. For higher values of $\mu$, the number of subdomains has been increased to improve the convergence. In particular, the equilibrium solutions for $\mu=2.184$ and $\mu=3.3$ have been obtained by using 200 sampling nodes. In these cases about 40 iterations are needed.

The image of Fig. $3 \mathrm{~b}$ and $3 \mathrm{c}$ are related to the cross section subject to the maximum bending moment $(s=L / 2)$ for the highest value of the multiplier $\mu=3.3$. Both figures refer to the deformed configuration, involving Eulerian functions. In Fig. 3b, the vertical diagram of $\left(\lambda_{z}(0, y, L / 2)-1\right)$ and the line with $\lambda_{z}(x, y, L / 2)=1$, which characterizes the points with no longitudinal deformation (the so called neutral axis for the deformation) are reported. As can be seen, both these lines are rectilinear. This according to the hypothesis of conservation of the planarity of the cross sections. On the basis of this hypothesis, in fact, the cross sections of the beam rotate rigidly (with a large angle) around the neutral axis. The values of the longitudinal stretch $\lambda_{z}$ at the ends of the vertical segment passing through the centroid are $\lambda_{z}=1.34998$ at $Y=H / 2$ and $\lambda_{z}=0.60938$ at $Y=-H / 2$. Similarly, the transversal stretches $\lambda_{x}=\lambda_{y}$ are worth $\lambda_{x}=\lambda_{y}=0.89595$ at $Y=H / 2$ and $\lambda_{x}=\lambda_{y}=1.11613$ at $Y=-H / 2$.

The longitudinal Cauchy stress $T_{3}$ is shown in the Fig. 3c for each point of the cross section by means of contour lines. The stress component $\mathrm{T}_{3}$ assumes the maximum tensile value at the upper edge, $\mathrm{T}_{3}=2.867$, and that of compression at the lower edge $T_{3}=-3.510$. Note that the neutral line for the stress $\left(\mathrm{T}_{3}=0\right)$ does not coincide with the neutral axis for the deformation $\left(\lambda_{z}=1\right)$.

The constitutive constants used in the application have been made dimensionless, dividing them by the constant $A_{2}$, to which the unit value was assigned. If the elastic constants are without dimensions, then, stresses are also such. Likewise, the geometrical dimensions of the beam have been rendered dimensionless, dividing them by the height $H$, which was assumed to have a unit value. In the same way, the variables $X, Y$ and $Z$ are normalized. Since these variables and stresses have been normalized, equilibrium equations (11) are also dimensionless and can therefore be compared with scalar zero. Evaluating therefore how much the three scalar equilibrium equations deviate from zero, it is possible to measure the accuracy of the solution obtained for the Euler beam.

Equilibrium equations are exactly satisfied at points belonging to the beam axis [52]. This is because for these points the displacements field is correct, being it kinematically compatible and equilibrated (system (15) has indeed been 
satisfied). On the other hand, when moving away from the beam axis, the equilibrium equations are no longer so well satisfied and some approximations appear as the edges of the cross sections are approached, since for these points the hypotheses underlying the kinematic model become less accurate.

In the light of the above, a numerical analysis was carried out, evaluating the equilibrium equations (11) at all points of the most stressed cross section ( $s=L / 2$ and $\mu=3.3$ ). Results are delivered in the diagrams illustrated by Figs. 3d, 3e and $3 \mathrm{f}$ for the equilibrium along the $X, Y$ and $Z$ axis, respectively. In these diagrams some contour lines, which join the points where the equations (11) give the same numeral values, are shown. As can be seen from Figs. 3d, 3e and $3 f$, even in the most critical case of the cross section subject to the maximum bending moment, the numerical values are very close to zero at each point of the cross section, showing that the solution obtained is actually accurate.

\section{Conclusions}

In the framework of fully nonlinear elasticity, the equilibrium problem of nonuniform bending of beams with compressible stored energy functions of polynomialtype has been formulated. By means of a kinematic model, which also takes into account the anticlastic inflexion of the cross sections, the three-dimensional displacement field of the beam has been described using four unknown functions. Three functions are needed to evaluate the rigid displacement of the cross sections. Two functions are the displacement components of the centroid of the generic cross section, while the third represents the rotation of such cross section. The anticlastic radius of curvature, which describes the inflexion of the cross section on its own plane, is the fourth unknown function.

The constitutive properties of material have been modeled through a stored energy function expressed as sum of powers of principal stretches with the addition of a convex function for the volume change. In this regard, some correlations formulae among the derivatives of the stored energy function with respect to the deformation invariants and those with respect to the principal stretches are established. Successively, the Piola-Kirchhoff stresses have been determined and the equilibrium conditions have been imposed. Specifically, the equilibrium equations have been written for the points belonging to the beam axis and for each individual cross section of the beam (in global form and not in the classic local form) equaling the internal bending moment, generated by the longitudinal Cauchy stresses, with the external bending moment, generated by external loads.

By assembling the derived formulae, the governing equations are obtained. These take the form of a coupled system of three equations in integral form, which is solved numerically through an iterative procedure. Solving this system, the displacement field has been obtained and then the shape assumed by the beam in the deformed configuration, the stretches and stresses in every point of the beam (following both Lagrangian and Eulerian descriptions) have been assessed. 
As numerical application, the finite bending of the Euler beam has been considered. Unlike the classic solution, the shape assumed by the deformed beam has been precisely determined and shown in a series of graphs, even in the cases with very high load multipliers. The vertical profiles of longitudinal stretch and stress for the deformed cross section, subject to the maximum bending moment, are displayed. Finally, the accuracy of the solution obtained has been estimated by checking a posteriori that the dimensionless equilibrium equations practically do not diverge locally from zero.

\section{Appendix. Expressions of the derivatives $S_{J, J}$}

In system (12) the derivatives $S_{J, J}=\frac{\partial S_{J}}{\partial J}$, for $J=X, Y, Z$ (no sum), assume the following forms: ${ }^{13}$

$$
\begin{aligned}
& S_{X, X}=2\left(\omega_{1, X}+I_{1, X} \omega_{2}+I_{1} \omega_{2, X}\right) \lambda_{X}-2 \omega_{2, X} \lambda_{X}^{3}+2\left(I_{3, X} \omega_{3}+I_{3} \omega_{3, X}\right) \frac{1}{\lambda_{X}} \\
& S_{Y, Y}=2\left(\omega_{1, Y}+I_{1, Y} \omega_{2}+I_{1} \omega_{2, Y}\right) \lambda_{Y}+2\left(\omega_{1}+I_{1} \omega_{2}\right) \lambda_{Y, Y}-2 \omega_{2, Y} \lambda_{Y}^{3} \\
& -6 \omega_{2} \lambda_{Y}^{2} \lambda_{Y, Y}+2 I_{3, Y} \omega_{3} \frac{1}{\lambda_{Y}}+2 I_{3} \omega_{3, Y} \frac{1}{\lambda_{Y}}-2 I_{3} \omega_{3} \frac{\lambda_{Y, Y}}{\lambda_{Y}^{2}} \\
& S_{Z, Z}=2\left(\omega_{1, Z}+I_{1, Z} \omega_{2}+I_{1} \omega_{2, Z}\right) \lambda_{Z}+2\left(\omega_{1}+I_{1} \omega_{2}\right) \lambda_{Z, Z}-2 \omega_{2, Z} \lambda_{Z}^{3} \\
& -6 \omega_{2} \lambda_{Z}^{2} \lambda_{Z, Z}+2 I_{3, Z} \omega_{3} \frac{1}{\lambda_{Z}}+2 I_{3} \omega_{3, Z} \frac{1}{\lambda_{Z}}-2 I_{3} \omega_{3} \frac{\lambda_{Z, Z}}{\lambda_{Z}^{2}}
\end{aligned}
$$

where $\omega_{i}=\frac{\partial \omega}{\partial I_{i}}, I_{i, K}=\frac{\partial I_{i}}{\partial K}$ and $\omega_{i, K}=\frac{\partial}{\partial K}\left(\frac{\partial \omega}{\partial I_{i}}\right)$ for $i=1,2,3, K=X, Y$, and with

$$
\begin{aligned}
& \lambda_{Y, Y}=\frac{\partial \lambda_{Y}}{\partial Y}=-\frac{1}{r(s)} e^{-\frac{Y}{r(s)}} \\
& \lambda_{Z, Z}=\frac{\partial \lambda_{Z}}{\partial Z}=r(s)\left[1-e^{-\frac{Y}{r(s)}} \cos \beta(s)\right] \frac{d^{2} \theta_{N}}{d s^{2}}, \\
& I_{1, X}=2 \lambda_{Z} \frac{1}{R(s)} e^{-\frac{Y}{r(s)}} \sin \beta(s), \\
& I_{1, Y}=-\frac{4}{r(s)} e^{-\frac{2 Y}{r(s)}}+2 \lambda_{Z} \frac{1}{R(s)} e^{-\frac{Y}{r(s)}} \cos \beta(s), \\
& I_{1, Z}=2 \lambda_{Z} r(s)\left[1-e^{-\frac{Y}{r(s)}} \cos \beta(s)\right] \frac{d^{2} \theta_{N}}{d s^{2}}, \\
& I_{3, X}=2 e^{-\frac{5 Y}{r(s)}} \lambda_{Z} \frac{1}{R(s)} \sin \beta(s), \\
& I_{3, Y}=-\frac{4}{r(s)} e^{-\frac{4 Y}{r(s)}} \lambda_{Z}^{2}+2 e^{-\frac{5 Y}{r(s)}} \lambda_{Z} \frac{1}{R(s)} \cos \beta(s), \\
& I_{3, Z}=2 \lambda_{Z} e^{-\frac{4 Y}{r(s)}}\left[1-e^{-\frac{Y}{r(s)}} \cos \beta(s)\right] \frac{d^{2} \theta_{N}}{d s^{2}} .
\end{aligned}
$$

${ }^{13}$ These derivatives have been used

$$
\begin{array}{ccc}
\frac{\partial \sin \theta_{N}(s)}{\partial s}=\frac{1}{R(s)} \cos \theta_{N}(s), & \frac{\partial \cos \theta_{N}(s)}{\partial s}=-\frac{1}{R(s)} \sin \theta_{N}(s), \\
\frac{\partial \sin \beta(s)}{\partial X}=\frac{1}{r(s)} \cos \beta(s), & \frac{\partial \cos \beta(s)}{\partial X}=-\frac{1}{r(s)} \sin \beta(s) .
\end{array}
$$




\section{Acknowledgement}

Financial support from the Italian Ministry of Education, University and Research (MIUR) in the framework of the Project PRIN2017 "Modelling of constitutive laws for traditional and innovative building materials" (code 2017HFPKZY) is gratefully acknowledged.

\section{References}

[1] Q. Wang, Y. Liu, Review of optical fiber bending/curvature sensor. Measurement 130 (2018) 161-176.

[2] B. Huang, M. Li, T. Mei, D. McCoul, S. Qin, Z. Zhao, J. Zhao, Wearable Stretch Sensors for Motion Measurement of the Wrist Joint Based on Dielectric Elastomers. Sensors 17 (2017) 2708, doi.org/10.3390/s17122708.

[3] J. Costa, F. Spina, P. Lugoda, L. Garcia, D. Roggen, N. Münzenrieder, Flexible Sensors-From Materials to Applications. Technologies 7 (2019) 35, doi:10.3390/technologies7020035.

[4] Z. Wang, Y. Yao, X. Wang, W. Yue, L. Chen, X. Xiang Zhangb, Bendinginduced electromechanical coupling and large piezoelectric response in a micromachined diaphragm. Sci Rep. 3 (2013) 3127, doi:10.1038/srep03127.

[5] G. Wang, M. Shahinpoor, Design, prototyping and computer simulations of a novel large bending actuator made with a shape memory alloy contractile wire. Smart Mater. Struct. 6 (1997) 214, doi.org/10.1088/0964$1726 / 6 / 2 / 011$.

[6] F. Schmitt, O. Piccin, L. Barbé, B. Bayle, Soft robots manufacturing: A review. Frontiers in Robotics and AI 5 (2018) 84, doi.org/10.3389/frobt.2018.00084.

[7] R.V. Martinez, J.L. Branch, C.R. Fish, L. Jin, R.F. Shepherd, R.M. Nunes, Z. Suo, G.M. Whitesides, Robotic tentacles with three-dimensional mobility based on flexible elastomers. Advanced materials 25 (2013) 205-212.

[8] B.C. Mac Murray, X. An, S.S. Robinson, I.M. Van Meerbeek, K.W. O'Brien, H. Zhao, R.F. Shepherd, Poroelastic foams for simple fabrication of complex soft robots. Advanced materials 27 (2015) 6334-6340.

[9] L. Wen, T. Wang, G. Wu, J. Liang, Quantitative thrust efficiency of a selfpropulsive robotic fish: Experimental method and hydrodynamic investigation. IEEE/ASME Transactions on Mechatronics 18 (2012) 1027-1038.

[10] P. Polygerinos, N. Correll, S.A. Morin, B. Mosadegh, C.D. Onal, K. Petersen, M. Cianchetti, M.T. Tolley, R.F. Shepherd, Soft robotics: Review of fluid-driven intrinsically soft devices; manufacturing, sensing, control, and 
applications in human-robot interaction. Advanced Engineering Materials 19 (2017) 1700016, doi.org/10.1002/adem.201700016.

[11] F. Ilievski, A.D. Mazzeo, R.F. Shepherd, X. Chen, G.M. Whitesides, Soft robotics for chemists. Angewandte Chemie International Edition 50 (2011) $1890-1895$.

[12] H. Wang, J. Ma, Z. Ren, Z. Gong, Y. Hao, T. Wang, L. Wen, Fiberreinforced soft robotic anthropomorphic finger. 2016 International Conference on Robotics and Automation Engineering (ICRAE). IEEE (2016)1-5.

[13] L.T. de Haan, J.M. Verjans, D.J. Broer, C.W. Bastiaansen, A.P. Schenning, Humidity-responsive liquid crystalline polymer actuators with an asymmetry in the molecular trigger that bend, fold, and curl. Journal of the American Chemical Society 136 (2014) 10585-10588.

[14] J. Zhao, A. Abbas, A low-cost soft coiled sensor for soft robots. ASME 2016 dynamic systems and control conference. American Society of Mechanical Engineers Digital Collection (2016).

[15] B.R. Seth, Finite Strain in Elastic Problems. Proc. R. Soc. Lond. A 234 (1935) 231-264.

[16] R.S. Rivlin, Large elastic deformations of isotropic materials. V. The problem of flexure. Proc. R. Soc. Lond. A 195 (1949) 463-473.

[17] J.L. Ericksen, Deformations possible in every isotropic, incompressible, perfectly elastic body. ZAMP J. of Appl. Math. and Phys. 5 (1954) 466-489.

[18] M.M. Carroll, Finite deformations of incompressible simple solids I. Isotropic solids. Quart. J. Mech. and Appl. Math. 21 (1968) 148-170.

[19] C.-C. Wang, Normal configurations and the nonlinear elastoplastic problems of bendings, torsion, expansion, and eversion for compressible bodies. Arch. Rational Mech. and Anal. 114 (1991) 195-236.

[20] R.W. Ogden, Non-linear elastic deformations, Ellis Horwood, Chichester (1984) and Dover Publications (1997).

[21] O.T. Bruhns, H. Xiao, A. Meyers, Finite bending of a rectangular block of an elastic Hencky material. J. Elasticity 66 (2002) 237-256.

[22] D.M. Haughton, Flexure and compression of incompressible elastic plates. Inter. J. of Eng. Scien. 37 (1999) 1693-1708.

[23] N. Triantafyllidis, Bifurcation phenomena in pure bending. J. Mech. Phys. Solids. 28 (1980) 221-245.

[24] C. Coman, M. Destrade, Asymptotic results for bifurcations in pure bending of rubber blocks. Quart. J. Mech. and Appl. Math. 61 (2008) 395-414. 
[25] S. Roccabianca, M. Gei, D. Bigoni, Plane strain bifurcations of elastic layered structures subject to finite bending: theory versus experiments. IMA J. of Appl. Math. 75 (2010) 525-548.

[26] A.E.H. Love, A treatise on the mathematical theory of elasticity. 4th Ed. Cambridge University Press, Cambridge (1927).

[27] R.T. Shield, Bending of a beam or wide strip. Quart. J. Mech. and Appl. Math. 45 (1992) 567-573.

[28] H. Lamb, Sur la flexion d'un ressort élastique plat. Philosophical Magazine 31 (1891) 182-188.

[29] T.M. Wang, S.L. Lee, O.C. Zienkiewicz, Numerical analysis of large deflections of beams. Inter. J. Mech. Scien. 3 (1961) 219-228.

[30] R. Frisch-Fay, Flexible bars. Butterworths, London (1962).

[31] T.M. Wang, Non-linear bending of beams with uniformly distributed loads. Inter. J. Nonlinear Mech. 4 (1969) 389-395.

[32] J.T. Holden, On the finite deflections of thin beams. Inter. J. Solid. Struct. 8 (1972) 1051-1055.

[33] K.-J. Bathe, S. Bolourchi, Large displacement analysis of three-dimensional beam structures. Num. Meth. Eng. 14 (1979) 961-986.

[34] J.C. Simo, A finite strain beam formulation. The three -dimensional beam structures. Part I. Comput. Meth. Appl. Mech. Eng. 49 (1985) 55-70.

[35] E. Reissner, On one-dimensional finite-strain beam theory: The plane problem. ZAMP J. of Appl. Math. and Phys. 23 (1972) 795-804.

[36] E. Reissner, On finite deformations of space-curved beams. ZAMP J. of Appl. Math. and Phys. 32 (1981) 734-744.

[37] J.C. Simo, L. Vu-Quoc, A three-dimensional finite-strain rod model. Part II: Computational aspects. Comput. Meth. Appl. Mech. Eng. 58 (1986) 79-116.

[38] J.C. Simo, L. Vu-Quoc, On the dynamics of flexible beams under large overall motions-The plane case: Part I. J. Appl. Mech. 53 (1986) 849-854.

[39] J.C. Simo, L. Vu-Quoc, On the dynamics of flexible beams under large overall motions-The plane case: Part II. J. Appl. Mech. 53 (1986) 855-854.

[40] A. Cardona, M. Geradin, A beam finite element non-linear theory with finite rotations. Int. J. Numer. Meth. Eng. 26 (1988) 2403-2438.

[41] J.C. Simo, L. Vu-Quoc, On the dynamics in space of rods undergoing large motions - a geometrically exact approach. Comput. Meth. Appl. Mech. Eng. 66 (1988) 125-161. 
[42] A. Ibrahimbegovič, On finite element implementation of geometrically nonlinear Reissner's beam theory: Three-dimensional curved beam elements. Comput. Methods Appl. Mech. Eng. 122 (1995) 11-26.

[43] L. Lanzoni. A.M. Tarantino. The bending of beams in finite elasticity. $J$. Elasticity 139 (2020) 91-121.

[44] F.O. Falope, L. Lanzoni, A. M. Tarantino, Bending device and anticlastic surface measurement of solids under large deformations and displacements. Mech. Res. Comm. 97 ( 2019) 52-56.

[45] A.M. Tarantino, L. Lanzoni, F.O. Falope, The bending theory of fully nonlinear beams. Springer (2019).

[46] F.O. Falope, L. Lanzoni, A. M. Tarantino, FE analyses of hyperelastic solids under large bending: The role of the Searle parameter and Eulerian slenderness. Materials MDPI 13(7) (2020) 1597. Published online 2020 Apr 1. doi: $10.3390 / \mathrm{ma} 13071597$

[47] L. Lanzoni. A.M. Tarantino. Mechanics of High-Flexible Beams under Live Loads. J. Elasticity (2020) doi.org/10.1007/s10659-019-09759-3.

[48] F.O. Falope, L. Lanzoni, A. M. Tarantino, The bending of fully nonlinear beams. Theoretical, numerical and experimental analyses. Inter. J. of Eng. Science. 145 (2019) 103167.

[49] R.W. Ogden, Large deformation isotropic elasticity: on the correlation of theory and experiment for incompressible rubberlike solids. Proc. R. Soc. Lond. A 326 (1972) 565-584.

[50] R.W. Ogden, Large deformation isotropic elasticity: on the correlation of theory and experiment for compressible rubberlike solids. Proc. $R$. Soc. Lond. A 328 (1972) 567-583.

[51] J.M. Ball, Convexity conditions and existence theorems in nonlinear elasticity. Arch. Ration. Mech. Anal. 63 (1977) 337-403.

[52] L. Lanzoni, A.M. Tarantino, Finite anticlastic bending of hyperelastic solids and beams. J. Elasticity 131 (2018) 137-170.

[53] J.M. Ciarlet, G. Geymonat, Sur les lois de comportement en élasticité nonlinéaire compressible. C.R. Acad Sci. Paris Sér. II 295 (1982) 423-426.

[54] A.M. Tarantino, Equilibrium paths of a hyperelastic body under progressive damage. J. Elasticity 114 (2014) 225-250.

[55] L. Lanzoni, A.M. Tarantino, Damaged hyperelastic membranes. Inter. J. Nonlinear Mech. 60 (2014) 9-22. 
[56] L. Lanzoni, A.M. Tarantino, Equilibrium configurations and stability of a damaged body under uniaxial tractions. ZAMP J. of Appl. Math. and Phys. 66 (2015) 171-190.

[57] L. Lanzoni, A.M. Tarantino, A simple nonlinear model to simulate the localized necking and neck propagation. Inter. J. Nonlinear Mech. 84 (2016) 94-104.

[58] M. Bacciocchi, A.M. Tarantino. Finite bending of hyperelastic beams with transverse isotropy generated by longitudinal porosity. European Journal of Mechanics/A Solids (2021). doi.org/10.1016/j.euromechsol.2020.104131

[59] M. Pelliciari, A.M. Tarantino, Equilibrium paths for Von Mises trusses in finite elasticity. J. Elasticity $\mathbf{5 7}$ (2019).

[60] M. Pelliciari, A.M. Tarantino, Equilibrium paths for Von Mises trusses in finite elasticity. Math. and Mech of Solids 25 (2020) 705-726.

[61] A.M. Tarantino, A. Nobili, Constitutive branching analysis of cylindrical bodies under in-plane equibiaxial dead-load tractions. Inter. J. Nonlinear Mech. 41 (2006) 958-968. 